\title{
Successful Laparoscopic Repair of Posttraumatic Diaphragmatic Injury
}

\author{
Khuram Khan ${ }^{1}$, Saqib Saeed ${ }^{2}$, Farhana Iqbal ${ }^{3}$, Leaque Ahmed ${ }^{2}$, Brian Donaldson ${ }^{4}$
}

1. Surgery, Columbia University College of Physicians and Surgeons, New York, USA 2. Surgery, Harlem Hospital Center, New York, USA 3. Internal Medicine, Richmond University Medical Center, Staten Island, USA 4. General Surgery, Columbia University College of Physicians and Surgeons, New York, USA

Corresponding author: Khuram Khan, khurram112@gmail.com

\begin{abstract}
Diaphragmatic injuries can be a direct result of penetrating thoracoabdominal trauma such as gunshot or stab wounds. Diaphragmatic rupture can lead to herniation of intra-abdominal organs into the thoracic cavity. Diagnosis can be difficult as the results of a physical exam can be unremarkable. A CT scan of the chest is diagnostic for diaphragmatic injuries. In most emergency cases, diaphragmatic injuries are managed with laparotomy where CT was diagnostic. We report a rare case of a 25-year-old man with right diaphragmatic injury sustained after a stab wound to the right liver managed successfully with laparoscopy.
\end{abstract}

Categories: Cardiac/Thoracic/Vascular Surgery, General Surgery, Trauma

Keywords: trauma, stab wound, laparoscopy, diaphragmatic injury, thoracoabdominal

\section{Introduction}

Diaphragmatic injuries (DIs) are rare but life-threatening conditions, and in most cases, they can be seen with both blunt and penetrating abdominal trauma. Diaphragmatic rupture after blunt thoracoabdominal trauma is seen in about $1 \%$ to $7 \%$ of cases, and in penetrating trauma, up to $15 \%$ of the cases [1]. Of these, about $8 \%$ of the cases where a patient sustained DI will need surgical interventions [2]. Management of such injuries with laparoscopy as an alternative to laparotomy has shown excellent results both diagnostically and therapeutically.

\section{Case Presentation}

A 25-year-old man with no prior medical history was transported to the hospital via the emergency medical service after he was stabbed with a seven-inch knife to the right thoracoabdominal area around the 10th intercostal space in the post axillary line. On arrival at the emergency department, he was alert, oriented, and reported right-sided abdominal pain. His vital signs were within normal limits, and oxygen saturation was $98 \%$ on room air. On physical examination, we noted a 6- to 7-cm jagged wound to the right

Received 09/25/2019

Review began 10/24/2019 Review ended 08/22/2020 Published 08/27/2020

(c) Copyright 2020

Khan et al. This is an open access article distributed under the terms of the Creative Commons Attribution License CC-BY 4.0., which permits unrestricted use, distribution, and reproduction in any medium, provided the original author and source are credited. thoracoabdominal area with profuse bleeding. The other results of his physical exam were unremarkable. No laboratory derangement was seen. Focused abdominal sonography for trauma and chest X-ray images were negative for bleeding and pneumothorax. Pressure dressing was applied to control bleeding from the wound. CT of the abdomen was obtained that revealed grade 3 hepatic laceration and no DIs (Figures 1, 2). 


\section{Cureus}

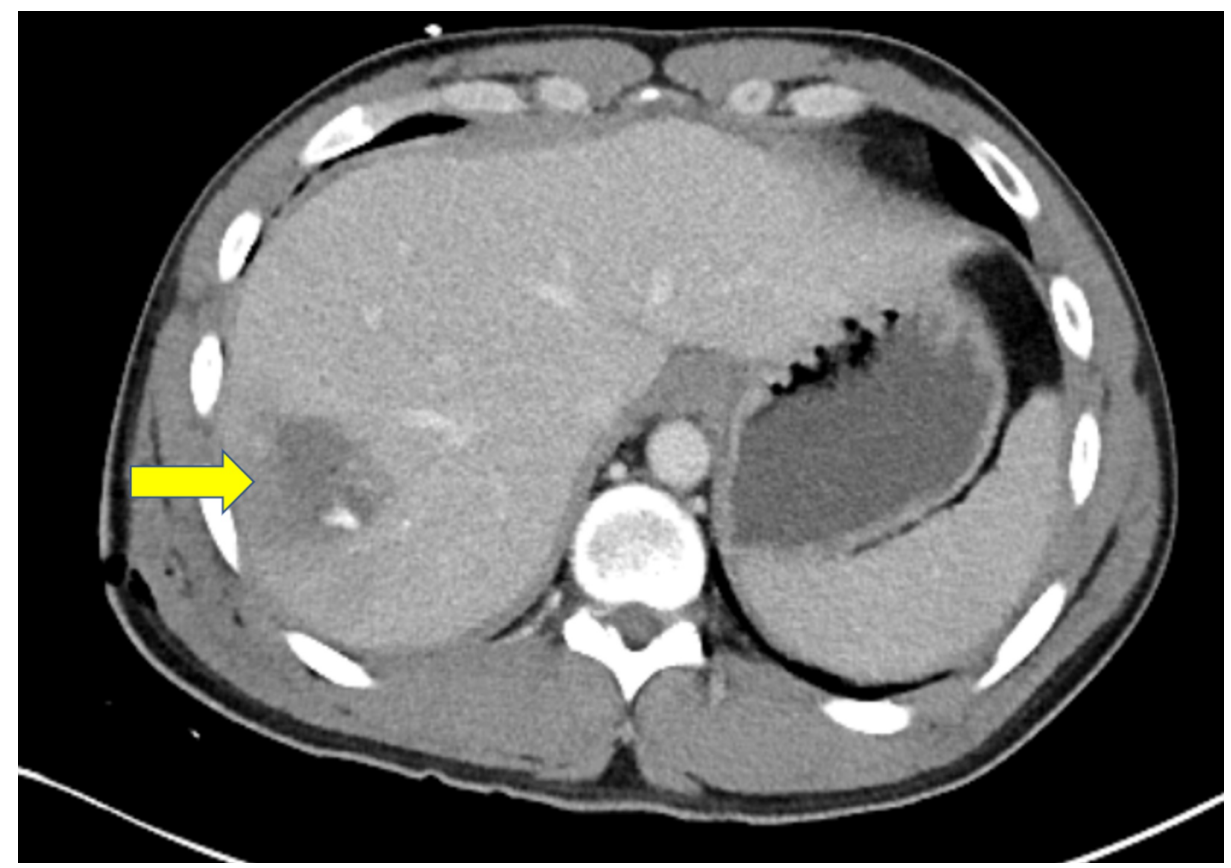

FIGURE 1: Abdomen CT, axial view, showing grade 3 hepatic laceration from knife stab wound (yellow arrow)

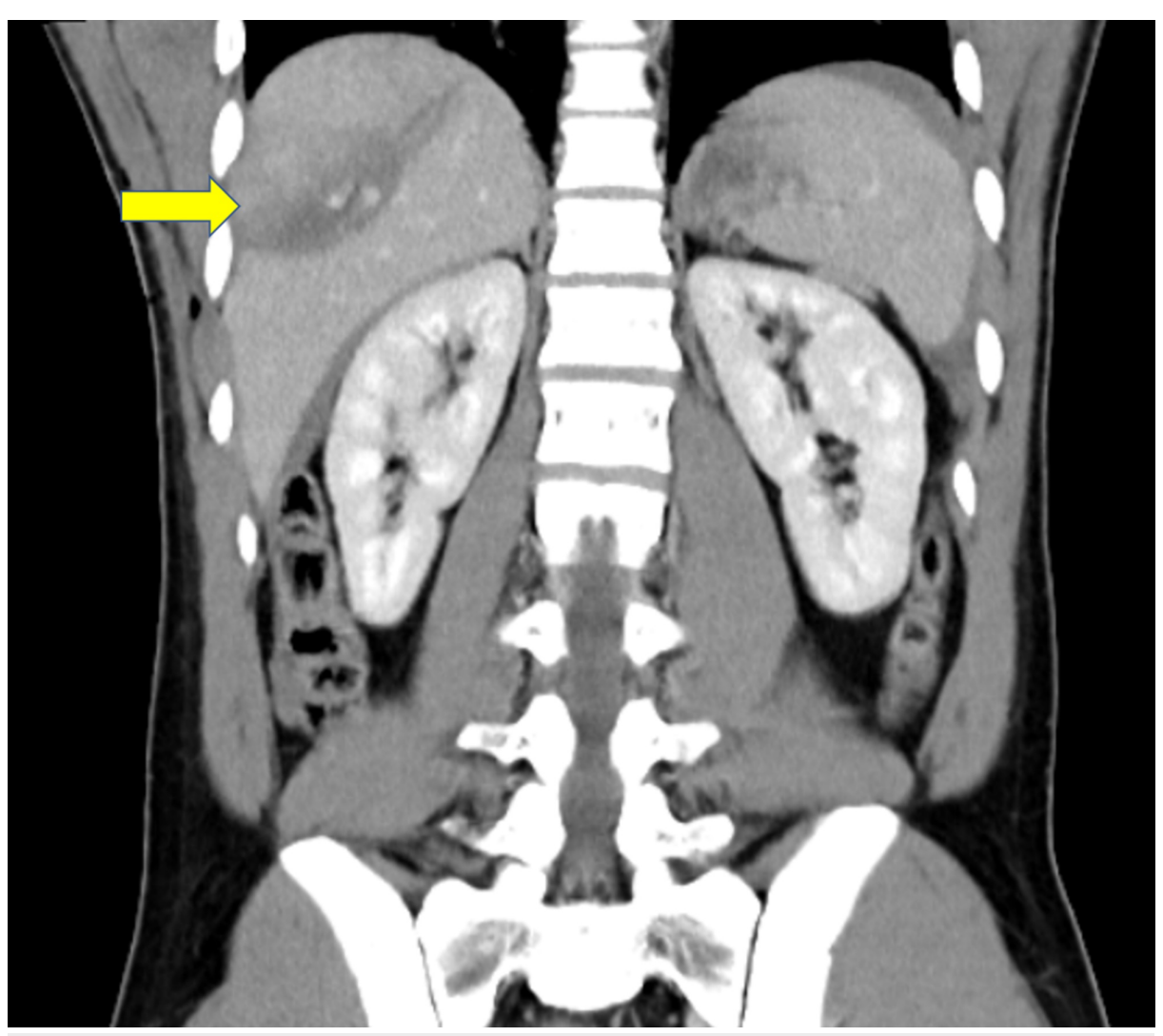

FIGURE 2: Abdomen CT, coronal view, showing grade 3 hepatic laceration after stab (yellow arrow) 


\section{Cureus}

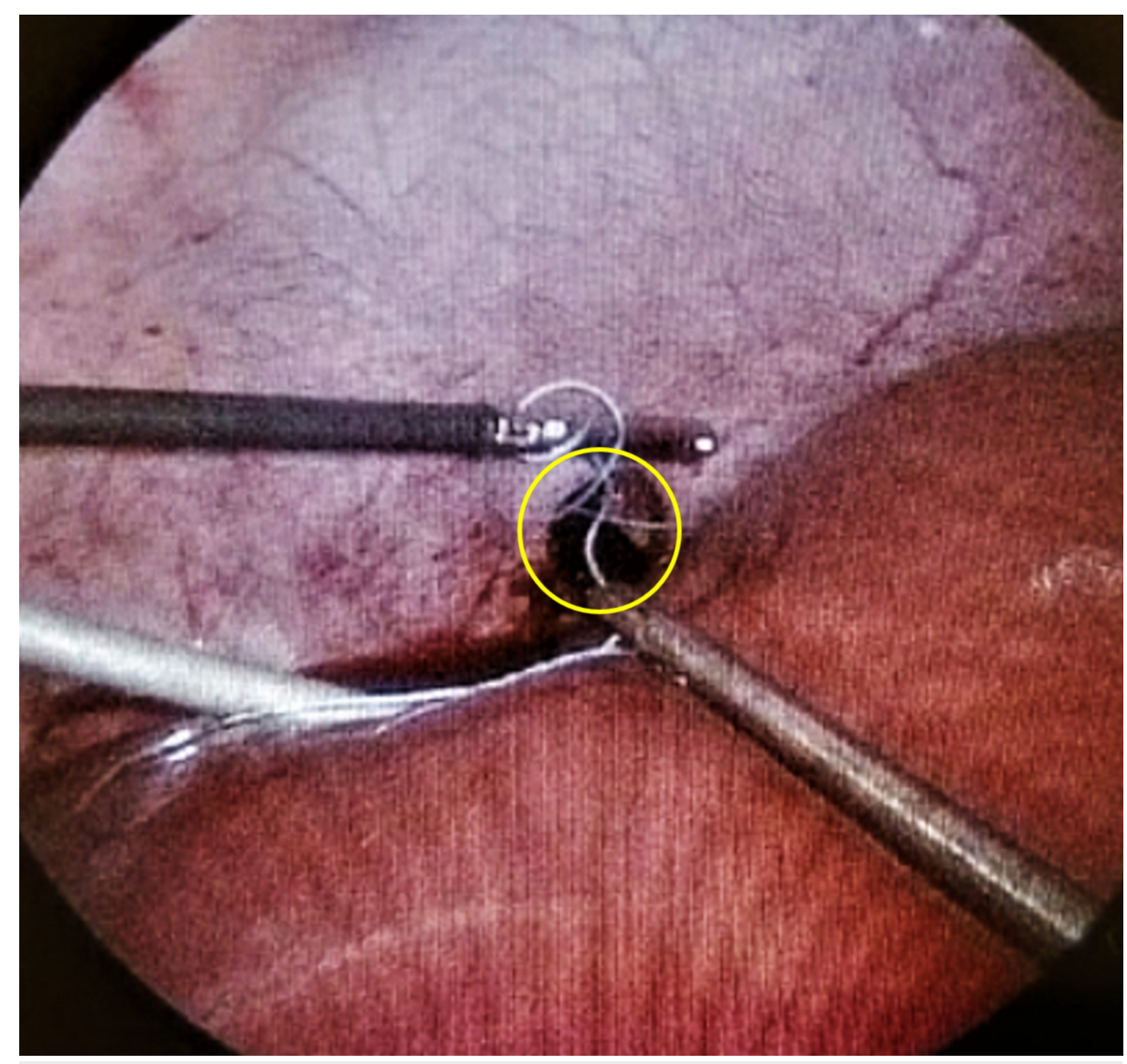

FIGURE 3: A 2-cm defect repaired via laparoscopy with suture (yellow circle outlines the defect) 


\section{Cureus}

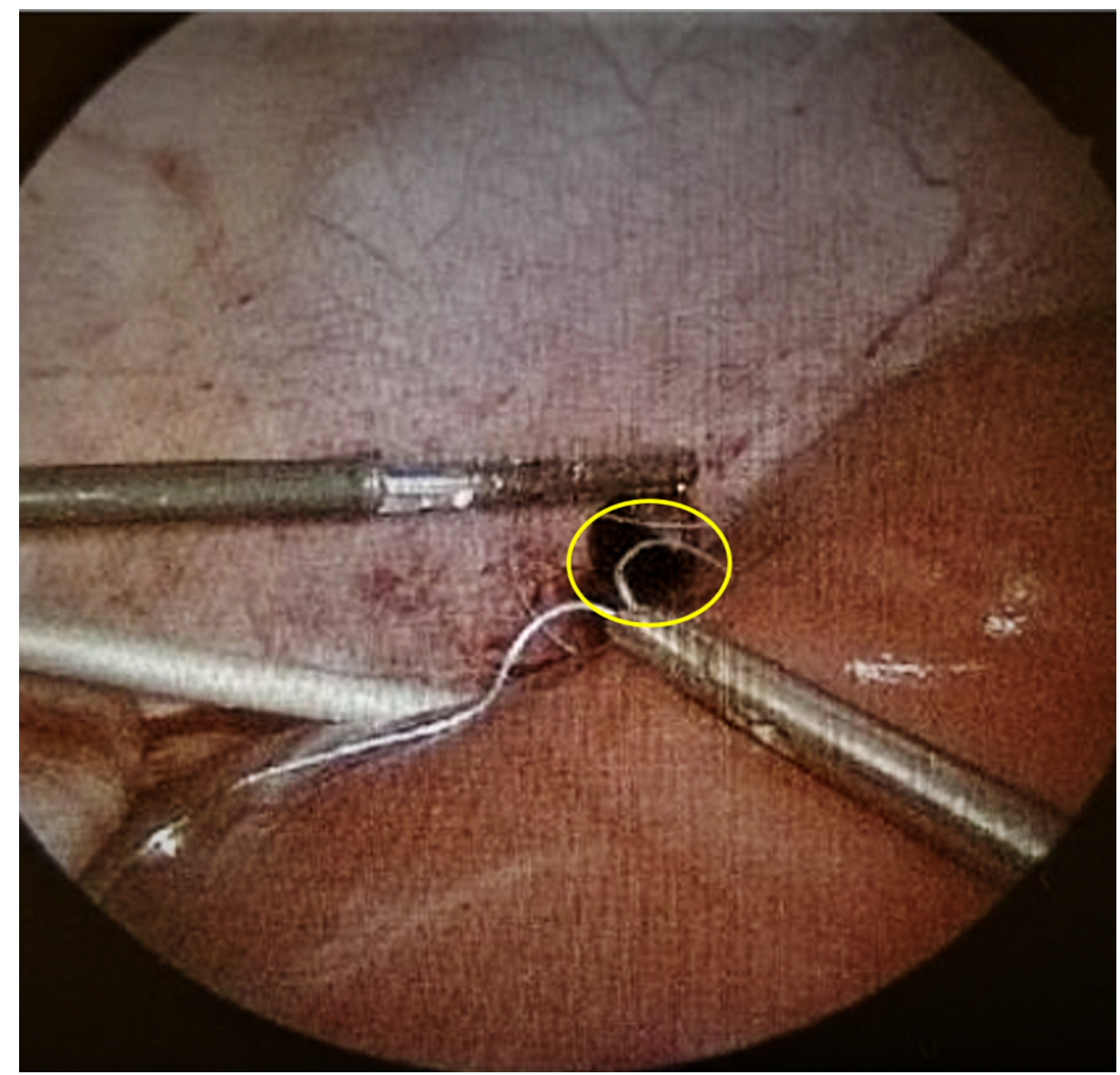

FIGURE 4: Repair of the diaphragm (yellow circle outlines defect)

Control of hepatic bleeding was achieved with hemostatic agents. The patient did well postoperatively, was extubated, and managed on the surgical floor. His chest tube was removed on postoperative day 4 , and the patient was discharged to home. On follow-up visit to the clinic, the patient was doing well and asymptomatic.

\section{Discussion}

DI is a rare and clinically underdiagnosed pathology directly resulting from blunt and penetrating thoracoabdominal trauma. Serious and life-threatening injuries can be seen with penetrating trauma and accounts for about $75 \%$ of cases [3]. One of the major challenges in successful management of a DI after trauma is diagnosing it early; correct diagnoses are often delayed, as in our case. Initial evaluation and imaging can miss most of the indicators of DI. Physical exam findings can be unremarkable, and imaging does not detect the injury that can be missed for months and even years after the initial injury [3].

In such cases, the initial evaluation in a hemodynamically stable patient can be done by obtaining a chest Xray followed by a CT scan of the chest and abdomen. CT scans can miss $30 \%$ to $50 \%$ of the diaphragmatic rupture during the initial assessment [4,5]. Interventions such as thoracoscopic and laparoscopic have sensitivity and specificity close to $100 \%$ for DIs. Choosing thoracoscopy versus laparoscopy depends on the skillset, stability of the patient, and indications for the surgery, e.g. retained hemothorax versus suspected intra-abdominal injury. Laparoscopy allows for a broader exploration of the entire diaphragm and peritoneal cavity. Laparoscopy also offers better intraoperative visualization of the diaphragm, which can contribute to better postoperative outcomes for the patient via reduction of pain, fewer respiratory complications, and reduced length of hospital stay [5]. DIs that are seen on left side of the diaphragm should undergo repair as the right diaphragm is well protected by the liver, but in rare case like in our patient you need to repair right side diaphragm injuries. Most DI cases can undergo primary repair where large defects require placement of a mesh.

\section{Conclusions}

Traumatic DIs can be life threatening and can be missed easily on initial evaluation after thoracoabdominal trauma. A high index of suspicion should prompt consideration of diagnostic laparoscopy to evaluate the diaphragm thoroughly in thoracoabdominal stab wounds. 


\section{Cureus}

\section{Additional Information}

\section{Disclosures}

Human subjects: Consent was obtained by all participants in this study. Conflicts of interest: In compliance with the ICMJE uniform disclosure form, all authors declare the following: Payment/services info: All authors have declared that no financial support was received from any organization for the submitted work. Financial relationships: All authors have declared that they have no financial relationships at present or within the previous three years with any organizations that might have an interest in the submitted work. Other relationships: All authors have declared that there are no other relationships or activities that could appear to have influenced the submitted work.

\section{References}

1. Mihos P, Potaris K, Gakidis J, et al.: Traumatic rupture of the diaphragm: experience with 65 patients . Injury. 2003, 34:169-172. 10.1016/S0020-1383(02)00369-8

2. Madden MR, Paull DE, Finkelstein JL, Goodwin CW, Marzulli V, Yurt RW, Shires GT: Occult diaphragmatic injury from stab wounds to the lower chest and abdomen. J Trauma. 1989, 29:292-298. 10.1097/00005373198903000-00003

3. Xenaki S, Lasithiotakis K, Andreou A, Chrysos E, Chalkiadakis G: Laparoscopic repair of posttraumatic diaphragmatic rupture. Report of three cases. Int J Surg Case Rep. 2014, 5:601-604. 10.1016/j.ijscr.2014.07.007

4. Pulido J, Reitz S, Gozdanovic S, Price P: Laparoscopic repair of chronic traumatic diaphragmatic hernia using biologic mesh with cholecystectomy for intrathoracic gallbladder. JSLS. 2011, 15:546-549. 10.4293/108680811X13176785204472

5. Lee PC, Lo C, Wu JM, Lin KL, Lin HF, Ko WJ: Laparoscopy decreases the laparotomy rate in hemodynamically stable patients with blunt abdominal trauma. Surg Innov. 2014, 21:155-165. $10.1177 / 1553350612474496$ 